\title{
Nucleosynthetic variations generated by size and density driven sorting of dust in protoplanetary disk
}

\author{
JEAN-DAVID BODÉNAN ${ }^{1}$, MARK A. HUTCHISON ${ }^{2}$, \\ LUCIO MAYER ${ }^{3}$ AND MARIA SCHÖNBÄCHLER ${ }^{4}$ \\ ${ }^{1}$ ETH Zürich/Universität Zürich \\ ${ }^{2}$ Ludwig-Maximilians-Universität München \\ ${ }^{3}$ Institute for Computational Science, Universität Zürich \\ ${ }^{4}$ ETH Zürich \\ Presenting Author: jean-david.bodenan@erdw.ethz.ch
}

Various isotopic systems studied in meteorites bear testimony of a nucleosynthetic heterogeneity in solar system bodies. As a first order observation, some systems show an isotopic dichotomy between objects formed in the inner solar system (non-carbonaceous chondrite reservoir) and those formed further out (carbonaceous chondrites reservoir). This can be attributed to the effects of a giant planet like Jupiter (e.g., [1]), although other models do not require a giant planet (e.g., [2]).

Here, we use the SPH code PHANTOM [3] with the MULTIGRAIN algorithm [4] to perform 3D gas and dust simulations of protoplanetary disks. They include 17 dust phases comprised of solar system silicate, presolar silicon-carbide, and presolar oxide grains. We use a homogeneous initial disk with or without a Jupiter-like planet. The surface densities were used in mass balance calculations to calculate the distributions of nucleosynthetic compositions across the disk in the different scenarios.

The presence of a planet creates a gap with pressure bumps on each side. These features can create changes in grain densities, particularly affecting the larger silicate grains. Our results show that size- and density-sorting generates differences in local abundances of presolar grains that could create isotopic heterogeneities in ${ }^{54} \mathrm{Cr}$, ${ }^{96} \mathrm{Zr}$, and ${ }^{50} \mathrm{Ti}$ in the order of those observed in solar system bodies. While the isotopic anomalies are essentially carried by the presolar grains, the final compositions are controlled by dilution with the larger solar system silicate grains.

\section{References}

[1] Alibert et al. (2018) Nature Astronomy 2, 873-877.

[2] Lichtenberg et al. (2021) Science 371, 365-370.

[3] Hutchison et al. (2018) Monthly Notices of the Royal Astronomical Society 476, 2186-2198.

[4] Mathis et al. (1977) The Astrophysical Journal 217, 425433. 